Pesq. Vet. Bras. 36(12):1155-1159, dezembro 2016 DOI: 10.1590/S0100-736X2016001200002

\title{
Effects of polysaccharide from Ophiopogon japonicus on immune response to Newcastle disease vaccine in chicken ${ }^{1}$
}

\author{
Xu Song ${ }^{2}$, Mei $\mathrm{Cao}^{3}$, Zhongqiong Yin ${ }^{2 *}$, Renyong Jia ${ }^{2,4}$, Yuanfeng Zou², Lixia $\mathrm{Li}^{2}$, \\ Guizhou Yue ${ }^{5}$, Xiaoxia Liang ${ }^{2}$, Lizi Yin² and Changliang $\mathrm{He}^{2}$
}

\begin{abstract}
Song X., Cao M., Yin Z., Jia R., Zou Y., Li L., Yue G., Liang X., Yin L. \& He C. 2016. Effects of polysaccharide from Ophiopogon japonicus on immune response to Newcastle disease vaccine in chicken. Pesquisa Veterinária Brasileira 36(12):1155-1159. Natural Medicine Research Center, College of Veterinary Medicine, Sichuan Agricultural University, Chengdu, 611130, China. E-mail: yinzhongq@163.com

In order to investigate the immune enhancement effects of Ophiopogon japonicus polysaccharide Ophiopogon japonicus (OJPS) on Newcastle disease (ND) live vaccine, chickens vaccinated against ND live vaccine was orally administered with the OJPS at high, medium and low concentrations respectively. In negative control group, chickens were given orally equal volume of physiological saline. On day 14, 21 and 28, the serum antibody titer, erythrocyte-C3b receptor rosette rate (E-C3bRR), erythrocyte-C3b immune complex rosette rate (E-ICRR) and peripheral lymphocyte proliferation were measured. The results showed that at most time points, the antibody titer, peripheral lymphocyte proliferation, E-C3bRR and elimination rate of immune complex of three OJPS administrating groups were significantly higher $(\mathrm{P}<0.05)$ than those in negative control group. It indicated that OJPS could significantly improve the immune efficacy of Newcastle disease live vaccine, Ophiopogon japonicus polysaccharide possessed synergistical immunoenhancement.
\end{abstract}

INDEX TERMS: Ophiopogon japonicus polysaccharide, antibody titer, peripheral lymphocyte proliferation, erythrocyte immunity, ND vaccine

\begin{abstract}
ABBREVIATIONS.- OJPS = polysaccharide extracted from Ophiopogon japonicus; $\mathrm{E}-\mathrm{C}_{3 \mathrm{~b}} \mathrm{RR}=$ erythrocyte- $\mathrm{C}_{3 \mathrm{~b}}$ receptor rosette rate; $\mathrm{E}$-ICRR = erythrocyte- $\mathrm{C}_{3 \mathrm{~b}}$ immune complex rosette rate; $\mathrm{ND}=$ Newcastle disease; $\mathrm{NDV}=$ Newcastle disease virus; $\mathrm{DMSO}=$ dimethyl sulfoxide; $\mathrm{CMF}=$ calcium and magnesium-free; PBS = phosphate-buffered saline; HI $=$ hemagglutination inhibition; MTT = 3-(4,5-dimethylthiazol-2-yl)-2,5-diphenyltetrazolium bromide; ConA = concanavalin A.
\end{abstract}

\footnotetext{
${ }^{1}$ Received on October 9, 2015.

Accepted for publication on June 25, 2016.

$\mathrm{Xu}$ Song, Mei Cao and Zhongqiong Yin contributed equally to this paper and should be considered as the first author.

${ }^{2}$ Natural Medicine Research Center, College of Veterinary Medicine, Sichuan Agricultural University, Chengdu, 611130, China. *Corresponding author: yinzhongq@163.com

3 Core Laboratory, Sichuan Provincial People's Hospital, Chengdu 610072, China.

${ }^{4}$ Key Laboratory of Animal Disease and Human Health of Sichuan Province, Sichuan Agricultural University, Chengdu, 611130, China.

${ }^{5}$ College of Science, Sichuan Agricultural University, Ya'an 625014, China.
}

\section{INTRODUCTION}

Newcastle disease (ND) resulting from infection with virulent Newcastle disease viruses (NDV) is one of the most important infectious diseases of poultry, and cause enormous economic loss and social disruption because of its worldwide distribution and high flock mortality (Miller et al. 2010, Ravindra et al. 2009). At present, there were no effective drugs to treat this disease and the main method to control ND was vaccination. Moreover, new strains of virus resistant to chemotherapy continue to emerge, so does the need for a safe and effective vaccine (Chen et al. 2010). The immune adjuvant can make vaccine generate a strong immune response providing long-term protection against infection (Chen et al. 2010). However, the benefits of adjuvant incorporation into a vaccine need to be balanced against the risk of adverse side effects, therefore, minimizing toxicity remains as one of the major challenges in adjuvant research (Chen et al. 2010). Polysaccharides as adjuvant are paving its way as a safe alternative.

Previous research found that polysaccharides could improve the immune response to ND vaccine in chicken, such 
as epimedium polysaccharides, astragalus polysaccharides, isatis root polysaccharides and so on (Chen et al. 2010, Fan et al. 2010, Kong et al. 2004).

Ophiopogon japonicus is a kind of traditional Chinese medicinal herb that is always used as a lung-moistening medicine. OJPS is the main active constituents of Ophiopogon japonicus, composed of $(1 \rightarrow 4)$-linked- $\alpha$-D-glucose (She $\&$ Shi 2003). It has been shown that OJPS possess anti-oxidative (Wang \& Luo 2008a; 2008b), immunomodulatory (Yu et al. 1991), anti-hypoxia (Xu \& Chen 1996), hypoglycemic (Qiu \& Li 2008), anti-myocardial ischemia (Zheng et al. 2007) activity.

In this study, the effects of Ophiopogon japonicus polysaccharide (OJPS) on serum antibody titer, peripheral lymphocyte proliferation, erythrocyte-C3b receptor rosette rate (E-C3bRR) and erythrocyte-C3b immune complex rosette rate (E-ICRR) were tested in chicken vaccinated with Newcastle disease (ND) vaccine taking the physiological saline as control. The purpose of this research was to observe whether Ophiopogon japonicus polysaccharide possessed synergistical immunoenhancement, and offer the theoretical evidence for developing potential new-type adjuvant.

\section{MATERIALS AND METHODS}

Extraction and purification of polysaccharides. The polysaccharide, OJPS, was prepared in our laboratory. Briefly, Ophiopogon japonicus was decocted 3 times with distilled water, the combined decoction was concentrated. Then total polysaccharide was extracted by ethanol precipitation $(\mathrm{v} / \mathrm{v}, 1: 3)$. The total polysaccharides was deproteinized with sevag's reagent, filtrated by chromatography on macroporous adsorption resins D101 and dialyzed against distilled water for 3 days in turn to remove pigment and other impurity. The content of polysaccharides was $86.82 \%$ measured by the phenol-sulfuric acid method (Li et al. 2009).

Material and reagent. RPMI-1640(Gibco) medium supplemented with benzylpenicillin $100 \mathrm{IU} / \mathrm{mL}$, streptomycin $100 \mathrm{IU} /$ $\mathrm{mL}$ and $10 \%$ Fetal bovine serum (Gibco) was used for washing, resuspending and culturing lymphocyte. ConA (Sigma), as T-lymphocyte mitogen, was dissolved into $0.1 \mathrm{mg} / \mathrm{mL}$ with RPMI- 1640 . The 3-(4, 5-dimethylthiazol-2-yl)-2,5-diphenyltetrazolium bromide (MTT, Amresco) and Sodium heparin was dissolved into $5 \mathrm{mg} /$ $\mathrm{mL}$ with phosphate buffered saline (PBS, $\mathrm{pH}$ 7.4). Lymphocytes separation medium (No. LTS1090) was bought from Haoyang Biological manufac-ture co., LTD, Tianjin, China.

Vaccine and virus. ND live vaccine (La Sota strain, No.100608) was offered by Liyuan Bio co., LTD, Guangxi, China. ND virus (La Sota strain) was supplied by Key laboratory of Animal Disease and Human Health of Sichuan Province, Sichuan Agricultural University, Ya'an, China.

Chickens and experimental design. Two hundred and three-yellow chickens at one day of age, purchased from Wens Co., Ltd Guangzhou, China, were randomly divided into four groups and reared in separated pens at $37^{\circ} \mathrm{C}$ with $24 \mathrm{~h}$ light during the first week, then gradually declined to $25^{\circ} \mathrm{C}$ and illumination time was reduced to $12 \mathrm{~h}$ per day. The chickens were fed with commercial chick starter and sufficient water.

On 7 days old, the average maternal serum hemaglutination inhibition (HI) antibody titer was less than $3 \log _{2}$, all chickens of each group (the average body weight (BW) was $120 \mathrm{~g}$ ) were vaccinated with ND live vaccine through nasal drip and eye-drop. At the same time, the chickens in three OJPS groups (high, medium and low doses of OJPS, OJPS ${ }_{H}, \mathrm{OJPS}_{\mathrm{M}}$ and OJPS ${ }_{\mathrm{L}}$ ) were orally administered with $0.5 \mathrm{~mL}$ of OJPS at concentrations of $100 \mathrm{mg} / \mathrm{kg}$ BW, $50 \mathrm{mg} / \mathrm{kg} \mathrm{BW}, 25 \mathrm{mg} / \mathrm{kg}$ BW respectively, once a day for five successive day, in negative control group (NC), with $0.5 \mathrm{~mL}$ of physiological saline, once a day for five successive day. On day 14,21 and 28 , six chickens were randomly selected from each group for determination of serum antibody titer by HI test; Another four chickens were randomly selected from each group for examination of E-C3bRR, E-ICRR by red cell yeast rosette formation test and peripheral lymphocyte proliferation by MTT method.

Serum HI antibody titer assay. Blood sample $(1 \mathrm{~mL}$ per chick) from jugular vein was collected and the serum was separated by centrifugation. After inactivated at $56^{\circ} \mathrm{C}$ for $30 \mathrm{~min}$, twofold serial dilution of serum, ranged from 1:2 to 1:2048, was added in a 96-well, V-shaped bottom microtiter plate containing $50 \mu \mathrm{L}$ of CMF-PBS in each well and then 50 $\mu \mathrm{L}$ of NDV antigen (4 HA units) was added into all wells except for the last row served as the controls. The antigen-serum mixture was incubated at $37^{\circ} \mathrm{C}$ for 10 min. Then, $50 \mu \mathrm{L}$ of $1 \%$ chicken erythrocytes suspension was added into each well and re-incubated for $30 \mathrm{~min}$. The condition of agglutination was monitored and recorded. The highest dilution of serum causing complete inhibition was considered the endpoint. The geometric mean titer was expressed as reciprocal log2 values of the highest dilution that displayed HI (Ma et al. 2010).

Peripheral lymphocyte proliferation assay. The peripheral lymphocyte, separated by lymphocyte separation medium from heart blood, was suspended with RPMI 1640 media (containing $10 \%$ fetal bovine serum) at a concentration of $5 \times 10^{6}$ cell $/ \mathrm{mL}$. $80 \mu \mathrm{L}$ of cell suspension and $20 \mu \mathrm{L}$ of Con A was added into each well of a 96-well microtiter plates, each sample seeded 4 wells. The plates were incubated at $39.5{ }^{\circ} \mathrm{C}$ with a humidified $5 \% \mathrm{CO}_{2}$. After incubation for $44 \mathrm{~h}, 20 \mu \mathrm{L}$ of MTT was added into each well, and the plates were re-incubated for another $4 \mathrm{~h}$. Then the plates were centrifuged at $1000 \mathrm{rpm}$ for $10 \mathrm{~min}$ to remove the supernatant carefully and $100 \mu \mathrm{L}$ of Dimethyl Sulfoxide were added into each well to dissolve the formazan crystal. The $\mathrm{OD}_{570 \mathrm{~nm}}$ was measured by an ELISA reader (Model 680, Bio-Rad Instruments) as the index of peripheral lymphocytes proliferation (Hung et al. 2010).

E-C3bRR and E-ICRR assay. Heparinized blood sample $(1 \mathrm{~mL}$ per chick) from jugular vein was centrifuged at $2000 \mathrm{rpm}$ for $5 \mathrm{~min}$ and erythrocytes were collected. After washed 3 times with Hanks' buffer (PH7.2), the erythrocytes were re-suspended with Hanks' buffer at a concentration of $1.25 \times 10^{7}$ cell $/ \mathrm{mL}$. $1 \%$ yeast suspension was prepared and boiled in thermostat water bath for $20 \mathrm{~min}$. After filtration, part of the yeast suspension was adjusted to $1 \times 10^{8} \mathrm{cell} / \mathrm{mL}$ and to be served as unsensitized yeast. The rest of yeast suspension was opsonized with an equal volume of fresh rat serum at $37^{\circ} \mathrm{C}$ with water bath for $20 \mathrm{~min}$ and then washed with Hank's buffer. The yeast was re-suspended in Hank's buffer at a concentration of $1 \times 10^{8} \mathrm{cell} / \mathrm{mL}$ and to be served as sensitized yeast. $50 \mu \mathrm{L}$ erythrocytes suspension were incubated with $50 \mu \mathrm{L}$ yeast suspension(sensitized yeast was used for measuring E-C3bRR, unsensitized yeast for measuring E-ICRR) at $37^{\circ} \mathrm{C}$ for $30 \mathrm{~min}$ by shaking periodically, then $100 \mu \mathrm{L}$ Hank's buffer and $25 \mu \mathrm{L} 0.25 \%$ glutaraldehyde were added and shaken gently and continually. 5 min later, three smears were prepared. After fixed with methanol and stained with Wright's stain, two hundred erythrocytes were counted from each smear under a microscope. E-C3bRR and E-ICRR = the number of erythrocytes binding to more than two yeast cells $/ 200 \times 100 \%$. The average percentage of three smears was calculated to obtain the results for each sample (Li et al. 2007). 
Statistical analyses. Data are shown as means \pm SD and provided to Statistical analyse using SPSS 17.0. Duncan's multiple range tests was used to evaluate the difference. Differences between means were considered significant at $\mathrm{p}<0.05$.

\section{RESULTS}

\section{The dynamic changes of antibody titer}

The dynamic changes of serum HI antibody titer were listed in Table.1. On day 14, the antibody titers in all OJPS groups were significantly higher than that in negative control group $(\mathrm{P}<0.05)$, and the antibody titer in medium dose of OJPS group was significantly higher than that in low dose of OJPS group. On day 28, no significant differences were observed in all groups. On day 28, the antibody titers in high and medium dose of OJPS groups were significantly higher than those in negative control group and low dose of OJPS group $(\mathrm{P}<0.05)$.

\section{The dynamic changes of peripheral lymphocyte proli- feration}

The dynamic changes of optical density in each group were shown in Table.2. On day 14, peripheral lymphocyte proliferation in all OJPS groups was significantly higher $(\mathrm{P}<0.05)$ than that in negative control group, and in high dose of OJPS group, the peripheral lymphocyte proliferation was significantly higher than those in medium and low dose of OJPS groups $(\mathrm{P}<0.05)$. On day 21 , the peripheral lymphocyte proliferation in all OJPS groups was significantly higher $(\mathrm{P}<0.05)$ than that in the negative control group; the high and medium dose of OJPS groups had higher effect on peripheral lymphocyte proliferation $(\mathrm{P}<0.05)$ in comparison with low dose of OJPS group. On day 28 , no significant differences were detected in the four experimental groups.

\section{The dynamic changes of $E-C_{3 b} R R$}

The dynamic changes of $E-C_{3 b} R R$ value in each group was listed in Table.3. On day 14, the $E-C_{3 b} R R$ values in all OJPS groups were significantly higher $(\mathrm{P}<0.05)$ than that in negative control group; significant differences were observed in the three OJPS groups, and the medium dose of OJPS group had best effect on $\mathrm{E}-\mathrm{C}_{3 \mathrm{~b}} \mathrm{RR}$. On day 21 , the $\mathrm{E}-\mathrm{C}_{3 \mathrm{~b}} \mathrm{RR}$ values in all CVPS groups were significantly higher than that in the negative control group, and in medium dose of OJPS group, the $E-C_{3 b} R R$ value was significant higher $(\mathrm{P}<0.05)$ than that in high and low dose of OJPS groups. On day 28, no significant differences were detected in the four experimental groups.

\section{The dynamic changes of E-ICRR}

The dynamic changes of E-ICRR value in each group was listed in Table.4. On day 14 and 28, the E-ICRR values in all OJPS groups were significantly lower $(\mathrm{P}<0.05)$ than that in the negative control group, and in high dose of OJPS group, the E-ICRR value was significantly lower $(\mathrm{P}<0.05)$ than those in medium and low dose of OJPS groups. On day 28 , the E-ICRR values in all OJPS groups were significantly lower $(\mathrm{P}<0.05)$ than that in negative control group, but no significant differences were observed in the three OJPS groups.
Table 1. The dynamic changes of serum $\mathrm{HI}$ antibody titer in each group $(\log 2)$

\begin{tabular}{lccc}
\hline Group & D14 & D21 & D28 \\
\hline OJPSH & $4.17 \pm 0.41^{\mathrm{bc}}$ & $4.50 \pm 0.55^{\mathrm{a}}$ & $4.50 \pm 0.55^{\mathrm{b}}$ \\
OJPSM & $4.67 \pm 0.52^{\mathrm{c}}$ & $4.67 \pm 0.81^{\mathrm{a}}$ & $4.67 \pm 0.52^{\mathrm{b}}$ \\
OJPSL & $3.83 \pm 0.75^{\mathrm{b}}$ & $4.67 \pm 0.52^{\mathrm{a}}$ & $3.83 \pm 0.41^{\mathrm{a}}$ \\
NC & $3.17 \pm 0.41^{\mathrm{a}}$ & $4.33 \pm 0.52^{\mathrm{a}}$ & $3.33 \pm 0.52^{\mathrm{a}}$
\end{tabular}

The superscripts within a column with different letters (a-c) differ significantly $(\mathrm{P}<0.05)$.

Table 2. The changes of peripheral lymphocyte proliferation in each group (OD570 value)

\begin{tabular}{lccc}
\hline Group & D14 & D21 & D28 \\
\hline OJPSH & $0.181 \pm 0.016^{\mathrm{c}}$ & $0.283 \pm 0.030^{\mathrm{c}}$ & $0.241 \pm 0.027^{\mathrm{a}}$ \\
OJPSM & $0.163 \pm 0.012^{\mathrm{bc}}$ & $0.276 \pm 0.023^{\mathrm{c}}$ & $0.233 \pm 0.016^{\mathrm{a}}$ \\
OJPSL & $0.144 \pm 0.012^{\mathrm{b}}$ & $0.218 \pm 0.024^{\mathrm{b}}$ & $0.229 \pm 0.022^{\mathrm{a}}$ \\
NC & $0.106 \pm 0.019^{\mathrm{a}}$ & $0.162 \pm 0.018^{\mathrm{a}}$ & $0.223 \pm 0.021^{\mathrm{a}}$
\end{tabular}

The superscripts within a column with different letters (a-c) differ significantly $(\mathrm{P}<0.05)$.

Table 3. The dynamic changes of E-C3bRR in each group

\begin{tabular}{lccc}
\hline Group & D14 & D21 & D28 \\
\hline OJPSH & $6.71 \pm 0.58^{\mathrm{c}}$ & $7.21 \pm 0.78^{\mathrm{b}}$ & $3.71 \pm 0.34^{\mathrm{a}}$ \\
OJPSM & $7.75 \pm 0.84^{\mathrm{d}}$ & $9.00 \pm 0.86^{\mathrm{c}}$ & $3.62 \pm 0.73^{\mathrm{a}}$ \\
OJPSL & $3.88 \pm 0.37^{\mathrm{b}}$ & $6.71 \pm 0.91^{\mathrm{b}}$ & $3.22 \pm 0.66^{\mathrm{a}}$ \\
NC & $2.75 \pm 0.29^{\mathrm{a}}$ & $3.12 \pm 0.48^{\mathrm{a}}$ & $3.00 \pm 0.41^{\mathrm{a}}$
\end{tabular}

The superscripts within a column with different letters (a-d) differ significantly $(\mathrm{P}<0.05)$.

Table 4. The changes of E-ICRR in each group

\begin{tabular}{lccc}
\hline Group & D14 & D21 & D28 \\
\hline OJPSH & $10.62 \pm 0.93^{\mathrm{c}}$ & $4.96 \pm 0.52^{\mathrm{c}}$ & $3.58 \pm 0.69^{\mathrm{b}}$ \\
OJPSM & $12.46 \pm 1.10^{\mathrm{b}}$ & $7.52 \pm 0.59^{\mathrm{b}}$ & $3.00 \pm 0.49^{\mathrm{b}}$ \\
OJPSL & $13.38 \pm 0.98^{\mathrm{b}}$ & $6.75 \pm 0.65^{\mathrm{b}}$ & $2.92 \pm 0.55^{\mathrm{b}}$ \\
NC & $15.12 \pm 1.07^{\mathrm{a}}$ & $10.12 \pm 0.96^{\mathrm{a}}$ & $6.74 \pm 0.50^{\mathrm{a}}$
\end{tabular}

The superscripts within a column with different letters (a-c) differ significantly $(\mathrm{P}<0.05)$.

\section{DISCUSSION}

The changes of serum antibody titer in poultry reflected the state of humoral immunity (Fan et al. 2010). Antibody usually appears within 6-10 days after infection in blood and local tissues (Rauw et al. 2009). The higher level of antibody titer has always been associated with better protection against ND (Huang et al. 2008). In the present study, the serum antibody titer in three 0JPS groups was almost higher at the three experimental time points in comparison with negative control group, the high and medium dosage of OJPS group possessed better activity in three OJPS groups. Treated with high and medium dosage of OJPS could induce the antibody titer rapidly rising to a high level in the earliest period and retain in the longest time. It indicated that the OJPS could accelerate the antibody production and improve the humoral immunity.

Both humoral immunity and cellular immunity play an important role in defense against NDV. In chickens, Cell-mediated immune responses play an important role to decrease the excretion and dissemination of the NDV (Rauw et al. 
2010). Lymphocytes proliferation is an important indicator reflecting the state of cellular immunity (Guo et al. 2009). In the present study, mitogen-induced lymphocyte proliferation in responses to the T-lymphocyte mitogen conA was significantly augmented in three OJPS groups with a dose-dependent manner in comparison with the control group on days 14 and 21. These results suggested that OJPS could promote the cellular immunity and the effect was strengthened gradually with the increase of dosage. The higher peripheral cellular immunity induced by OJPS might be involved in a greater proportion of specific T-lymphocytes (Guo et al. 2009). Similar results had been observed in astragalus polysaccharide that orally administration for three days could enhance the NDV antibody levels in chickens (Chen et al. 2010).

The immune function of erythrocytes play the major carriers of immune complexes(IC) in the circulation via their complement receptor(E-C3b, CR1), these erythrocytes bind $\mathrm{C} 3 \mathrm{~b}$ bearing immune complexes and transport them to the organs of the monocyte phagocytic system and reticuloendothelial system where the IC were eliminated, including the liver and spleen (Zhou et al. 2010, Gershon 1997). Defect of E-C3b could lead to tissue injury by IC which escaped from the red cell immune system (Jiang et al. 2010). In this study, the E-C3bRR of chickens treated with OJPS was always higher than that of negative control group on days 14 and 21, and the medium dosage of OJPS group possessed better activity on E-C3bRR in three OJPS groups, thus it suggested that OJPS could increase the activity of CR1, and enhance the expression of E-C3b (Gershon 1997).

After vaccinated against ND vaccine, ND-specific antibodies were abundantly produced by chick immune organs and many circulating immune complexes (CIC) were formed in blood circulation. The CIC were adhered by erythrocytes via CR1 causing the E-ICRR rising, then quickly carried to be cleaned up (Li et al. 2007, Yoshida et al. 1986). In our research, the E-ICRR in all four experimental groups was reached a higher level after vaccination, and then it went down gradually. On day 14, the E-ICRR in high dosage of OJPS group was significantly lower than that in negative control group, and it was turned to normal level on day 21, while the medium and low dosage of OJPS groups were recovered on day 28. At the same time, the negative control group was still higher on days 21 and 28 than the three OJPS groups. These results demonstrated the OJPS could accelerate clearing the CIC in a dose dependent way. This was agreement with the studies in Selenium, Sodium Selenite and epimedium polysaccharides (Deng et al. 1999, Luo et al. 2009, Yang et al. 2000, 2001).

The correlation of immunoenhancement efficacy with the dosage of OJPS was also investigated in this research. Our observations shown that different dosage of OJPS possessed a dose-dependent relationship in peripheral lymphocyte proliferation and E-ICRR, however this relationship did not exist in serum antibody titer and E-C3bRR. Similar effects of some Chinese herbal medicinal ingredients on serum antibody titer and epimedium polysaccharides on E-C3bRR in chickens immunized with ND vaccine has been reported(Luo et al. 2009, Wang et al. 2005).

\section{CONCLUSIONS}

The OJPS not only improved the E-C3bRR and accelerated the elimination rate of CIC, but also induced higher antibody titer and peripheral lymphocyte proliferation in chickens vaccinated against ND live vaccine.

These results confirmed that OJPS could enhance the immune response of ND vaccine in chicken, and it would be expected as a candidate of new-type immunologic adjuvant.

Acknowledgements.- This study was supported by the Foundation for the Author of Excellent Doctoral Dissertation of Sichuan Agricultural University (YB2014003), the International cooperation projects of Sichuan Province (2014HH0058, 2013HH0042), the Sichuan Youth Science and Technology Innovation Research Team for waterfowl disease prevention and control (2013TD0015) and the National Natural Science Foundation of China (Grant no.31372477).

Contribution of the authors.- Conceived and designed the experiments: $\mathrm{Xu}$ Song, Mei Cao, Zhongqiong Yin, Renyong Jia. Performed the experiments: Xu Song, Mei Cao, Yuanfeng Zou. Analyzed the data: Yuanfeng Zou, Lixia Li, Lizi Yin, Changliang He. Contributed reagents/materials/analysis tools: Lixia Li, Xiaoxia Liang, Guhou Yue. Wrote the paper: Xu Song.

\section{REFERENCES}

Chen Y.K., Wang D.Y., Hu Y.L., Guo Z.H., Wang J.M., Zhao X., Fan Y.P., Guo L.W., Yang S.J., Sai F.D. \& Xing Y.J. 2010. Astragalus polysaccharide and oxymatrine can synergistically improve the immune efficacy of Newcastle disease vaccine in chicken. Int. J. Biol. Macromol. 46:425-428.

Deng H., Yang H., Liu Y.Q. \& He Y.M. 1999. Effect of sodium selenite on the humoral immunity and erythrocyte immune function of chick vaccinated against NDV by adding to drink water. Chinese J. Vet. Sci. Technol. 7:21-22.

Fan Y.P., Hu Y.L., Wang D.Y., Guo Z.H., Zhao X.N., Guo L.W., Zhao B., Zhang J. \& Wang Y.L. 2010. The Luong Nguyen epimedium polysaccharide and propolis flavone can synergistically stimulate lymphocyte proliferation in vitro and enhance the immune responses to ND vaccine in chickens. Int. J. Biol. Macromol. 47:87-92.

Gershon H. 1997. The anti-inflammatory role of the erythrocyte: impairment in the elderly. Arch. Gerontol. Geriatr. 24:157-165.

Guo Z.H., Hu Y.L., Wang D.Y., Ma X., Zhao X.N., Zhao B.K., Wang J.M. \& Liu P. 2009. Sulfated modification can enhance the adjuvanticity of lentinan and improve the immune effect of ND vaccine. Vaccine 27:660-665.

Huang X.Y., Hu Y.L., Zhao X.N., Lu Y., Wang J.M., Zhang F. \& Sun J.L. 2008. Sulfated modification can enhance the adjuvant activity of astragalus polysaccharide for ND vaccine. Carbohydr. Polym. 73:303-308.

Hung C., Yeh C., Chen H., Lai C., Kuo M., Yeh M., Lin W., Tu M., Cheng H. \& Chen C. 2010. Porcine lactoferrin administration enhances peripheral lymphocyte proliferation and assists infectious bursal disease vaccination in native chickens. Vaccine 28:2895-2902.

Jiang J.B., Wu C.H., Gao H., Song J.D. \& Li H.Q. 2010. Effects of Astragalus polysaccharides on immunologic function of erythrocyte in chickens infected with infectious bursa disease virus. Vaccine 28:5614-5616.

Kong X.F., Hu Y.L., Rui R., Wang D.Y. \& Li X.R. 2004. Effects of Chinese herbal medicinal ingredients on peripheral lymphocyte proliferation and serum antibody titer after vaccination in chicken. Int. Immunopharmacol. 4:975-982.

Li F., Yuan Q.P. \& Rashid F. 2009. Isolation, purification and immunobiological activity of a new water-soluble bee pollen polysaccharide from Crataegus pinnatifida Bge. Carbohydr. Polym. 78:80-88.

Li H., Lloyd R. \& Wang J. 2007. Effect of Astragalus polysaccharides on erythrocyte immune adherence of chickens inoculated with infectious bursa1 disease virus. Agricult. Sci. China 11:1402-1408.

Luo Y., Shao Y.B., Gu X.L. \& Li X.Y. 2009. Effects of epimedium herb polysaccharides on the immunity function and vaccination in chickens. China Poultry 1:22-26. 
Ma X., Guo Z.H., Wang D.Y., Hu Y.L. \& Shen Z.Q. 2010. Effects of sulfated polysaccharides and their prescriptions on immune response of ND vaccine in chicken. Carbohydr. Polym. 82:9-13.

Miller P.J., Decanini E.L. \& Afonso C.L. 2010. Newcastle disease: evolution of genotypes and the related diagnostic challenges. Infect. Genet. Evol. 10:26-35.

Qiu B.H. \& Li R.M. 2008. Effects of polysaccharide in Ophiopogon japonicus on blood glucose in gestational diabetic rats. J. Jinan Univ. (Med. Ed.) 4:367-369.

Rauw F., Gardin Y., Palya V., Anbari S., Lemaire S., Boschmans M., Berg T. \& Lambrecht B. 2010. Improved vaccination against Newcastle disease by an in ovo recombinant HVT-ND combined with an adjuvanted live vaccine at day-old. Vaccine 28:823-833.

Rauw F., Gardin Y., Palya V., Borm S., Gonze M., Lemaire S., Berg T. \& Lambrecht B. 2009. Humoral, cell-mediated and mucosal immunity induced by oculo-nasal vaccination of one-day-old SPF and conventional layer chicks with two different live Newcastle disease vaccines. Vaccine 27:3631-3642.

Ravindra P.V., Tiwari A.K., Ratta B., Baisa M.V., Chaturvedi U., Palia S.K., Bhaskar S. \& Chauhan R.S. 2009. Time course of Newcastle disease virus-induced apoptotic pathways. Virus Res. 144:350-354.

She G.M. \& Shi J.P. 2003. Study on structural features of two neutral polysaccharides Md-1 and Md-2 from Ophiopogon japonicus. Northwest Pharmaceut. J. 2:58-59.

Wang D.Y., Hu Y.L., Sun J.L., Kong X.F., Zhang B.K. \& Liu J.G. 2005. Comparative study on adjuvanticity of compound Chinese herbal medicinal ingredients. Vaccine 23:3704-3708.
Wang Z.J. \& Luo D.H. 2008a. Isolation, purification and antioxidant activity study of Radix ophiopogonis water-soluble polysaccharide OPA. Modern Traditional Chinese Medicine 5:77-79.

Wang Z.J. \& Luo D.H. 2008b. Isolation, purification and antioxidant activity study of Radix ophiopogonis water-soluble polyaccharide OPB. J. Changchun Univ. Traditional Chinese Medicine 3, 254-255.

Xu Y.P. \& Chen Q. 1996. Ophiopogon japonicus polysaccharide protection against cerebrum ischaemia. J. Zhenjiang Medical College 3:217-218.

Yang H., Deng H., Liu Y.Q. \& He Y.M. 2000. Effect of Selenium on the erythrocyte immune function of chick. J. Foshan Univ. (Nat. Sci. Ed.) 4:65-68.

Yang H., Deng H., Liu Y.Q. \& He Y.M. 2001. Effect of Selenium on the erythrocyte immune Adherence and body weight of chick vaccinated against NDV. Chinese J. Vet. Drug 4:18-20.

Yoshida K., Yukiyama Y. \& Miyamoto T. 1986. Interaction between immune complexes and C3b receptors on erythrocytes. Clin. Immunol. Immunopathol. 39:213-221.

Yu B.Y., Yin X. \& Zhang C.H. 1991. The immune activity of Ophiopogon japonicus polysaccharides. J. China Pharmaceut. Univ. 5:286-288.

Zheng Q., Feng Y., De D.S. \& Chen Y.Z. 2007. Protective effect of Ophiopogonis polysaccharide MDG-1 on experimental myocardial ischemic rats. Chinese J. Integrated Traditional and Western Medicine 12:11161120.

Zhou J.Q., Pan Q., Wang T., Chen C.C. \& Cai B.Y. 2010. Effects of Codonopsis pilosula polysaccharide on antibody titer and lymphocytes proliferation in chicken immunized with ND vaccine. Jiangsu Agricult. Sci. 3:263-264. 